\title{
Assembly and annotation of a non-model gastropod (Nerita melanotragus) transcriptome: a comparison of De novo assemblers
}

\author{
Shorash Amin ${ }^{1}$, Peter J Prentis ${ }^{2}$ Edward K Gilding ${ }^{3}$ and Ana Pavasovic ${ }^{1 *}$
}

\begin{abstract}
Background: The sequencing, de novo assembly and annotation of transcriptome datasets generated with next generation sequencing (NGS) has enabled biologists to answer genomic questions in non-model species with unprecedented ease. Reliable and accurate de novo assembly and annotation of transcriptomes, however, is a critically important step for transcriptome assemblies generated from short read sequences. Typical benchmarks for assembly and annotation reliability have been performed with model species. To address the reliability and accuracy of de novo transcriptome assembly in non-model species, we generated an RNAseq dataset for an intertidal gastropod mollusc species, Nerita melanotragus, and compared the assembly produced by four different de novo transcriptome assemblers; Velvet, Oases, Geneious and Trinity, for a number of quality metrics and redundancy.

Results: Transcriptome sequencing on the lon Torrent PGM ${ }^{\mathrm{TM}}$ produced 1,883,624 raw reads with a mean length of 133 base pairs (bp). Both the Trinity and Oases de novo assemblers produced the best assemblies based on all quality metrics including fewer contigs, increased N50 and average contig length and contigs of greater length. Overall the BLAST and annotation success of our assemblies was not high with only 15-19\% of contigs assigned a putative function.
\end{abstract}

Conclusions: We believe that any improvement in annotation success of gastropod species will require more gastropod genome sequences, but in particular an increase in mollusc protein sequences in public databases. Overall, this paper demonstrates that reliable and accurate de novo transcriptome assemblies can be generated from short read sequencers with the right assembly algorithms.

Keywords: Nerita melanotragus, De novo assembly, Transcriptome, Heat shock protein, lon torrent

\section{Background}

The phylum Mollusca is a highly abundant group of marine animals accounting for over $23 \%$ of all marine species $[1,2]$, and as such are a dominant taxa of many marine ecosystems. These organisms are also of significant economic importance as a source of bioactive compounds in addition to being aquaculture and fisheries commodities. Molluscs also serve as valuable models for behavioural neurobiology, respiration and feeding in animals [3-6]. Consequently, molluscs are very important both economically and ecologically. However, genomic resources remain scarce for Mollusc species, with transcriptome data

\footnotetext{
* Correspondence: a.pavasovic@qut.edu.au

'School of Biomedical Sciences, Faculty of Health, Queensland University of Technology, GPO Box 2434, Brisbane, Qld 4001, Australia

Full list of author information is available at the end of the article
}

availble for only select species such as Crassostrea gigas, Macoma balthica, Aplysia californica and Lymnaea stagnalis [7-10] As a result, this group of organisms remains relatively poorly studied at the genomic level.

Research into the genomics of gastropod molluscs has lagged, because genomic resources are not developed for many species. Next generation sequencing platforms such as Illumina and Ion Torrent have recently been used to rapidly characterise transcriptome sequences from a number of non-model organisms [11-14]. In this study, we use the Ion Torrent platform, an efficient and low cost platform to sequence the Nerita melanotragus transcriptome, a non-model species without a reference genome.

Precise and accurate de novo assembly and annotation of transcriptomes, however, is a commonly overlooked but critically important step for assemblies generated 
from short reads ( 100-150 bp). Recently, many de novo assemblers have been developed with specific algorithms for transcriptome assembly from Illumina short reads, nonetheless their effectiveness for de novo assembly of Ion Torrent data remains relatively unexplored as the Ion Torrent technology is newer and still gaining acceptance in the research community. Accurate assembly of short reads into longer contigs is important for the functional annotation of ESTs in non-model organisms. In fact, one of the major challenges for genomic research in mollusc species is that many genes remain unannotated. In this study, we address these issues by comparing the performance of a number of short read de novo transcriptome assemblers using Ion Torrent sequence data in Nerita melanotragus.

The black nerite ( $N$. melanotragus) is a marine gastropod within the phylum Mollusca. This species inhabits the intertidal zone and has a large geographic distribution from central Queensland, Australia to southern New Zealand [15]. As a consequence, the environmental conditions that this species is exposed to change temporally and spatially, on both micro and macro geographic scales. Thus, this organism is a good candidate to explore the genetic and gene expression changes, which allow it to persist in such a dynamic environment. Little is known about adaptation genetics and plastic gene expression changes in $N$. melanotragus due an explicit lack of genomic resources. To address this issue, we report a first de novo assembly of the N. melanotragus transcriptome. Specifically, this study focuses on addressing the following aims: 1. to generate genomic resources for this species through whole organism transcriptome sequencing; and 2. to assess the accuracy and precision of four different short read de novo transcriptome assemblers.

\section{Methods}

Black nerite ( $N$. melanotragus), (Figure 1A), individuals were collected from the rocky intertidal zone at Caloundra, Queensland, Australia (26 $48^{\prime} 17^{\prime \prime} \mathrm{S} 153^{\circ} 8^{\prime}$ $\left.28^{\prime \prime} \mathrm{E}\right)$. Ethics approval and collection permits/licenses were not required for specimen collection. Individual

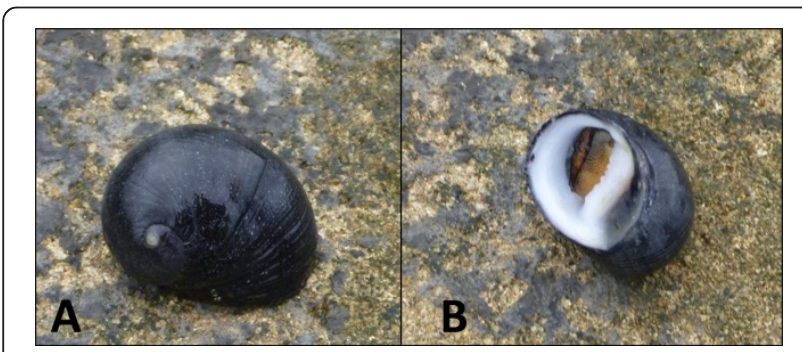

Figure 1 Black nerite (N. melanotragus). Black nerite displaying external morphology (A) and black nerite displaying tan/brown colouration of its operculum (B). animals were classified as $N$. melanotragus based on operculum colour [16]. A single individual was snap frozen in liquid nitrogen $\left(\mathrm{LN}_{2}\right)$ and stored at $-80^{\circ} \mathrm{C}$ until RNA extraction. The frozen tissue sample from the whole organism was homogenised in $\mathrm{LN}_{2}$ and total RNA was extracted using a Trizol/Chloroform extraction protocol followed by a clean up using an RNeasy Minikit (Qiagen). RNA samples were treated using Turbo DNase (Ambion), according to manufacturer's protocol.

To check the quantity and integrity of the total RNA, the sample was run on a Bioanalyzer 2100 RNA Nano chip (Agilent Technologies). Messenger RNA was isolated from total RNA using the Dynabeads mRNA Purification Kit (Life Technologies). A Bioanalyzer 2100 Pico chip (Agilent Technologies) was used to determine the quality and quantity of isolated mRNA.

High quality mRNA (100-500 ng) was fragmented into 200-700 bp pieces using RNase III (Life Technologies) and Agincourt beads were used to remove small RNA fragments. The yield and size distribution of fragmented RNA was determined on a Bioanalyzer 2100 using a RNA 6000 Pico chip (Agilent Technologies). Library construction was conducted as per the Ion Total RNA-Seq Kit (Life Technologies) for whole transcriptome libraries and cDNA yield and size was determined using a Bioanalyzer 2100 high sensitivity DNA chip.

Template preparation for sequencing was conducted according to the OneTouch Ion $^{\mathrm{max}}$ Template Kit (Life Technologies). Ion Torrent sequencing was conducted using the Ion PGM ${ }^{\mathrm{TM}} 200$ Sequencing Kit (Life Technologies) on an Ion Torrent Personal Genome Machine $\left(\mathrm{PGM}^{\mathrm{TM}}\right.$, Life Technologies) using a 318-chip (Ion $318^{\mathrm{TM}}$ chip, Life Technologies).

Raw sequencing reads were converted to FastQ files and assessed for quality scores. Reads were accepted based on a quality threshold $(\mathrm{Q}>20$, ambiguous bases less than $1 \%)$, and adapter sequences were removed prior to downstream analyses. To critically assess the quality of this Ion Torrent data we undertook a number of analytical approaches described by [17] including the sequencing depth and coverage for expressed genes from the publically available mitochondrial genome of $N$. melanotragus using Geneious Pro (Version 5.6) [18].

High quality reads were assembled into contiguous sequences (contigs) using four different short read de novo assemblers, which included following: 1) Geneious Pro (Version 5.6) [18]; 2) Velvet, short read assembler, Version 1.2.08 [19]; 3) Oases short read assembler, Version 0.2.08 [20] and 4) Trinity short read assembler [21]. All assemblers except Geneious used the following assembly parameters: kmer hash length $=25$, coverage cut-off $=3 x$; minimum contig length $=100 \mathrm{bp}$. In the Geneious software, kmer hash length and coverage cut-off could not be changed, so default settings were used with a minimum 
contig length of $100 \mathrm{bp}$. The assembly created by the four different assemblers were compared for three different parameters; the number of contigs produced, the N50 statistic and the longest contig to determine which assembler performed best.

To determine the redundancy of the assemblies produced by the four different assemblers we remapped our assembled datasets to the mitochondrial reference gene set from $N$. melanotragus (publically available from NCBI). All contigs produced by the four different assemblers were remapped to this gene set and the overall number of hits was calculated as a quality score.

Following contig generation, the transcriptome assemblies for Trinity and Oases were referenced to the NR database at NCBI as BLASTx queries using the Blast2 $\mathrm{GO}^{\circ}$ software suite [22]. In order to be used in downstream analyses, BLASTx hits had to be below an E-value of $1 \times 10^{-6}$. Annotation analyses were performed at levels 2 and 3 . The Blast $2 \mathrm{GO}^{\circ}$ software suite was also used to predict the functions of contigs with BLASTx hits and assign Gene Ontology (GO) terms to the sequences. To determine which of the short read assemblers produced the best assembly of our Ion Torrent data, we compared the BLAST and annotation success of these different datasets.

To validate the reliability and accuracy of our assembly and annotation, we randomly chose two contigs (annotated as $\beta$ (beta) - actin and NADH dehydrogenase subunit 5) and designed primers for PCR and Sanger sequencing. Primers were designed using BatchPrimer3 (Version 1.0) using settings as per [23]. Details of the primer sequences are provided in the supplementary material for this paper (Additional file 1: Table S1). PCR was performed according to the MyTaq ${ }^{\mathrm{TM}}$ (Bioline) protocol with the following concentrations of reagents $1 \times \mathrm{PCR}$ Buffer, $1 \mu \mathrm{M}$ of each primer, 0.1 units of MyTaq ${ }^{\mathrm{TM}}$ DNA Polymerase (Bioline) and $20 \mathrm{ng}$ of template genomic DNA (from same individual that was sequenced) in a total volume of $25 \mu \mathrm{L}$. PCR conditions were as follows: $3 \mathrm{~min}$ at $94^{\circ} \mathrm{C}$, followed by 30 cycles of $30 \mathrm{sec}$ at $94^{\circ} \mathrm{C}, 30 \mathrm{sec}$ at $52^{\circ}$ C, $30 \mathrm{sec} 72^{\circ} \mathrm{C}, 3 \mathrm{~min}$ at $72^{\circ} \mathrm{C}$. Amplicons were purified using the Isolate PCR Kit (Bioline) and cycle sequencing was carried out using BigDye ${ }^{\circ}$ Terminator v3.1 Cycle Sequencing Kit (Life Technologies). After a $\mathrm{MgSO}_{4}$ cleanup, the amplicons were run on an ABI 3500 Genetic Analyzer (Life Technologies). Sequences were visualised and edited by eye using Geneious Pro Version 5.6. These sequences were then used as BLASTn queries against the nucleotide database at NCBI and were compared for differences against the original sequences.

\section{Results}

lon torrent sequencing and reads assembly

Transcriptome sequencing of mRNA from N. melanotragus on the Ion Torrent PGM platform generated a total of $249.67 \mathrm{Mbp}$ of sequence from 1,883,624 raw reads. Mean length of reads was $133 \mathrm{bp}$, with the longest read being $392 \mathrm{bp}$. Sequence reads that did not meet our strict quality criteria $(\mathrm{Q}<20$, ambiguous bases $>1 \%)$ were excluded and $84.19 \mathrm{Mb}$ of high quality data was retained for downstream analysis, as low quality bases are likely to reduce the accuracy of transcriptome assemblies.

Based on high quality reads, a total of 112762,78 306, 10886 and 3090 contigs were generated using the following four different assemblers Geneious, Velvet, Trinity and Oases, respectively (Table 1 ). Overall the Oases assembly produced the longest contig at $1700 \mathrm{bp}$ closely followed by Trinity at $1618 \mathrm{bp}$. The longest contigs produced by Geneious and Velvet were over $700 \mathrm{bp}$ shorter than the other two de novo assemblers (Table 1). The length of the N50 statistic in the Geneious, Velvet and Oases assemblies were noticeably shorter than that calculated for the Trinity assembly (Table 1). Average contig length showed a similar trend, with the Trinity assembly also having the longest average contig length.

Both Velvet and Geneious assemblies had a greater number of contigs remapped to the mitochondrial expressed gene set with 450 and 420 hits respectively, compared to 37 and 25 hits for Trinity and Oases, respectively. The coverage of the contigs produced by both Trinity and Oases was greater than $95 \%$, while the coverage produced by the Geneious and Velvet contigs was less than $55 \%$ for both assemblies. The Geneious and Velvet assemblies were found to be highly redundant and produced more fragmented contigs, consequently they were removed from further analyses.

Remapping of high quality reads to the transcribed genes in $N$. melanotragus mitochondrial genome resulted in an assembly with an average of approximately $374 \times$ read depth and greater than $99.5 \%$ coverage. The sequencing depth was highest for the $16 \mathrm{~S}$ rRNA gene with $>2000 \times$ read depth. All genes had coverage of greater than $98 \%$ with the lowest coverage occurring in NADH dehydrogenase subunit 2 , which contained a $40 \mathrm{bp}$ region with no coverage.

Table 1 Assembly quality metrics

\begin{tabular}{lllll}
\hline Assembly Statistic & \multicolumn{3}{l}{ Assembler } \\
\cline { 2 - 5 } & Oases & Trinity & Velvet & Geneious \\
\hline Number of contigs & 3090 & 10886 & 78306 & 112762 \\
Average contig length & 175 & 293 & 111 & 140 \\
Longest contig & 1700 & 1618 & 458 & 711 \\
N50 & 149 & 258 & 107 & 124 \\
\hline
\end{tabular}

Assembly statistics for the transcriptomes produced by the four different short read de novo assemblers. 


\section{Functional annotation of contigs}

Of the 10886 and 3090 contigs queried against the NR database only 2069 and 475 returned significant hits at greater than $1 \times 10^{-6}$ stringency (Table 2). This meant that approximately 19 and $15.4 \%$ of contigs could be assigned putative functions for the Trinity and Oases assemblies, respectively.

Despite the limited number of contigs assigned BLAST hits, the contigs generated by both Trinity and Oases captured a broad range of different types of transcripts, as indicated by the variety of Gene Ontology (GO) terms assigned. A total functional annotation dataset is provided in (Additional file 1: Figure S1 and Figure S2), and here we report only the results for the top 20 GO terms for each category (Figure 2). The GO category with the highest number of terms assigned molecular function, followed by cellular component while biological process had the least contigs assigned terms. The most commonly assigned GO terms in the molecular function GO category were the housekeeping genes involved in ATP binding, protein binding and structural constituent of ribosome for both assemblies (Figure 2). Oxidation-reduction process, translation and translational elongation were the most commonly assigned terms for the biological process GO category. The three most commonly assigned GO terms for cellular component were cytosol, cytoplasm and nucleus, and cytoplasm, integral to membrane and mitochondrion for the Trinity and Oases assembly, respectively. Over $65 \%$ of BLAST hits were made up of different mollusc species. The Pacific oyster, C. gigas, which made up 27 and 34\% of BLAST hits for the Oases and Trinity assemblies, dominated top BLAST hits. Other molluscs including Haliotis discus and $H$. diversicolor were also in the top four species that made up top BLAST hits for both assemblies (Figure 3).

\section{PCR validation of contigs}

The PCR primer pairs designed for beta-actin and NADH dehydrogenase subunit 5 amplified a single product of the correct size, images provided in the

Table 2 Annotation results

\begin{tabular}{lcc}
\hline Annotation category & \multicolumn{2}{c}{ Annotation result (number of sequences) } \\
\cline { 2 - 3 } & Trinity & Oases \\
\hline Without blast result & $0(0 \%)$ & $0(0 \%)$ \\
Without blast hits & $8823(81 \%)$ & $2615(84.6 \%)$ \\
With blast result & $301(2.7 \%)$ & $66(2.1 \%)$ \\
With mapping result & $177(1.6 \%)$ & $28(0.9 \%)$ \\
Annotated sequences & $1585(14.5 \%)$ & $381(12.3 \%)$ \\
Total sequences & 10886 & 3090 \\
\hline
\end{tabular}

The number of contigs allocated to different annotation categories for the Trinity and Oases assemblies.
(Additional file 1: Figure S1). High quality sequence was obtained for both amplicons using both forward and reverse primers. The BAC and NAD sequences were assigned top nucleotide blast hits for beta-actin from Aplysia californica and NADH dehydrogenase subunit 5 from $N$. melanotragus, respectively. Protein blast confirmed this result with an E-value of greater than $1 \times 10^{-27}$. Alignment of the beta-actin and NADH dehydrogenase subunit 5 sequences to the contigs from which they were designed resulted in a perfect match for beta-actin and the presence of a single one base pair indel for NADH dehydrogenase subunit 5 , in an adenosine homopolymer region.

\section{Discussion}

The availability and throughput of next generation sequencing technologies has enabled the rapid and efficient sequencing of transcriptomes for model and nonmodel species. The majority of de novo transcriptome assemblies in non-model organisms have in the past been produced using the long reads (300-600 bp) generated using Roche 454 [24]. With the recent developments in sequencing technology, short read sequencers (90-400 bp), such as Illumina and Ion Torrent, are starting to be more commonly used for the generation of large next generation sequencing data sets, as the costs are much lower for the same output [25]. Consequently, the use of short read sequencers to generate de novo transcriptome assemblies for non-model organisms may lead to a more complete gene set for these species at a lower cost. The reliability of de novo transcriptome assemblies generated from short read sequencers, however, needs to be validated to ensure that assemblies are accurate and won't compromise the downstream applications of next generation sequencing projects. In this paper we compare a number of de novo assemblers to demonstrate that short read RNA-seq data generated by an Ion Torrent $\mathrm{PGM}^{\mathrm{TM}}$ sequencing system can reliably and accurately be assembled for a non-model organism.

Accurate de novo assembly of transcriptomes is crucial for next generation sequencing projects in non-model organisms. Of particular importance is finding short read assembly algorithms that produce accurate and reliable assemblies from the short reads produced by Ion Torrent or Illumina sequencers. In our comparison of four different short read assemblers using Ion Torrent data, we found that Trinity and Oases outperformed Velvet and Geneious in all performance metrics, including longer N50 and average contig lengths, producing fewer and longer contigs and having less redundant contigs. Overall, these results are similar to those obtained when comparing Trinity or Oases against other short read assemblers in simulation studies and empirically, with Illumina data [26]. Even though Trinity and Oases outperformed the other assemblers in all metrics, their respective assemblies 


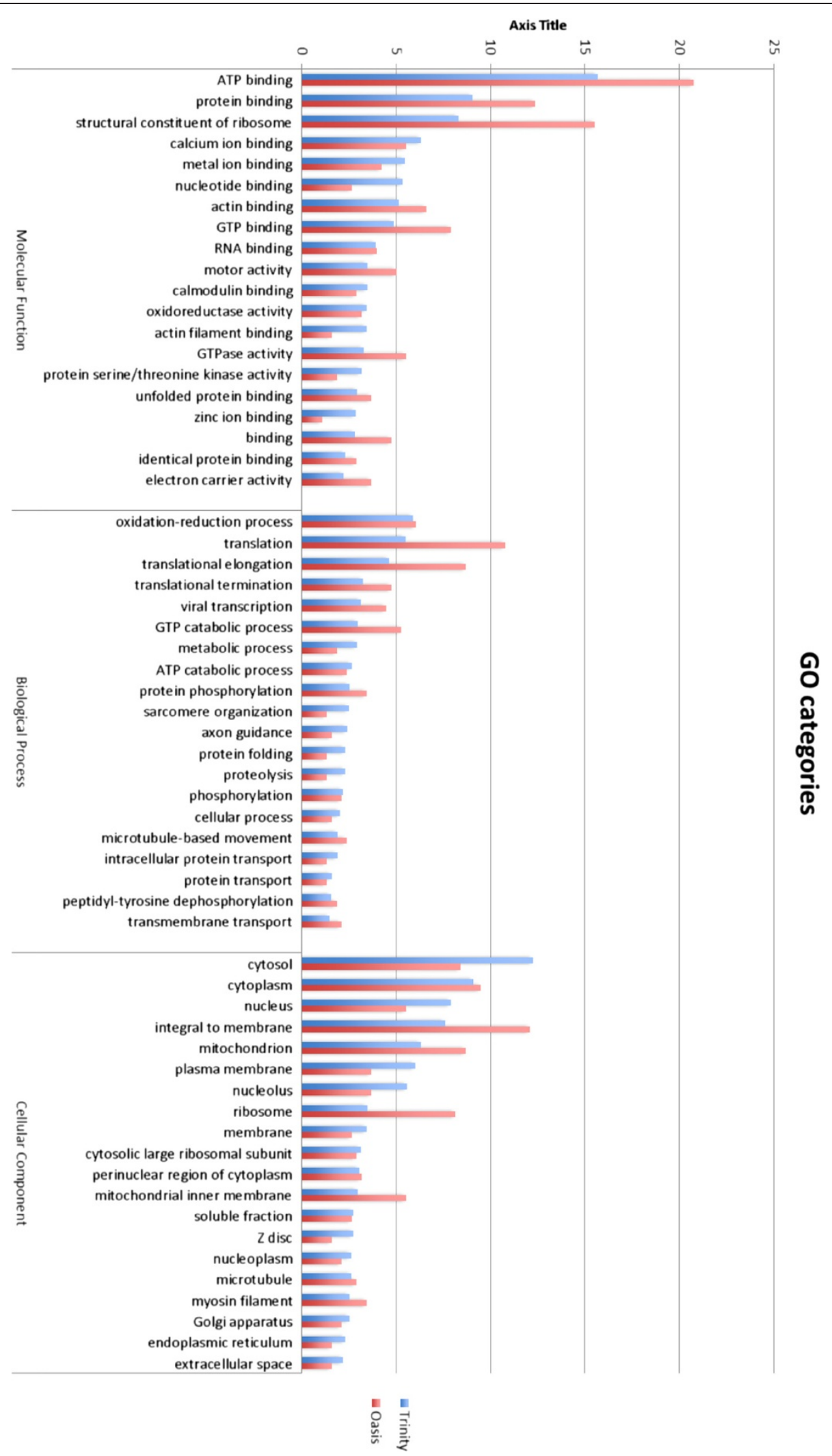

Figure 2 GO category assignment. Comparative analysis and functional classification of the top $20 \mathrm{GO}$ terms for the Trinity and Oases assembly. 


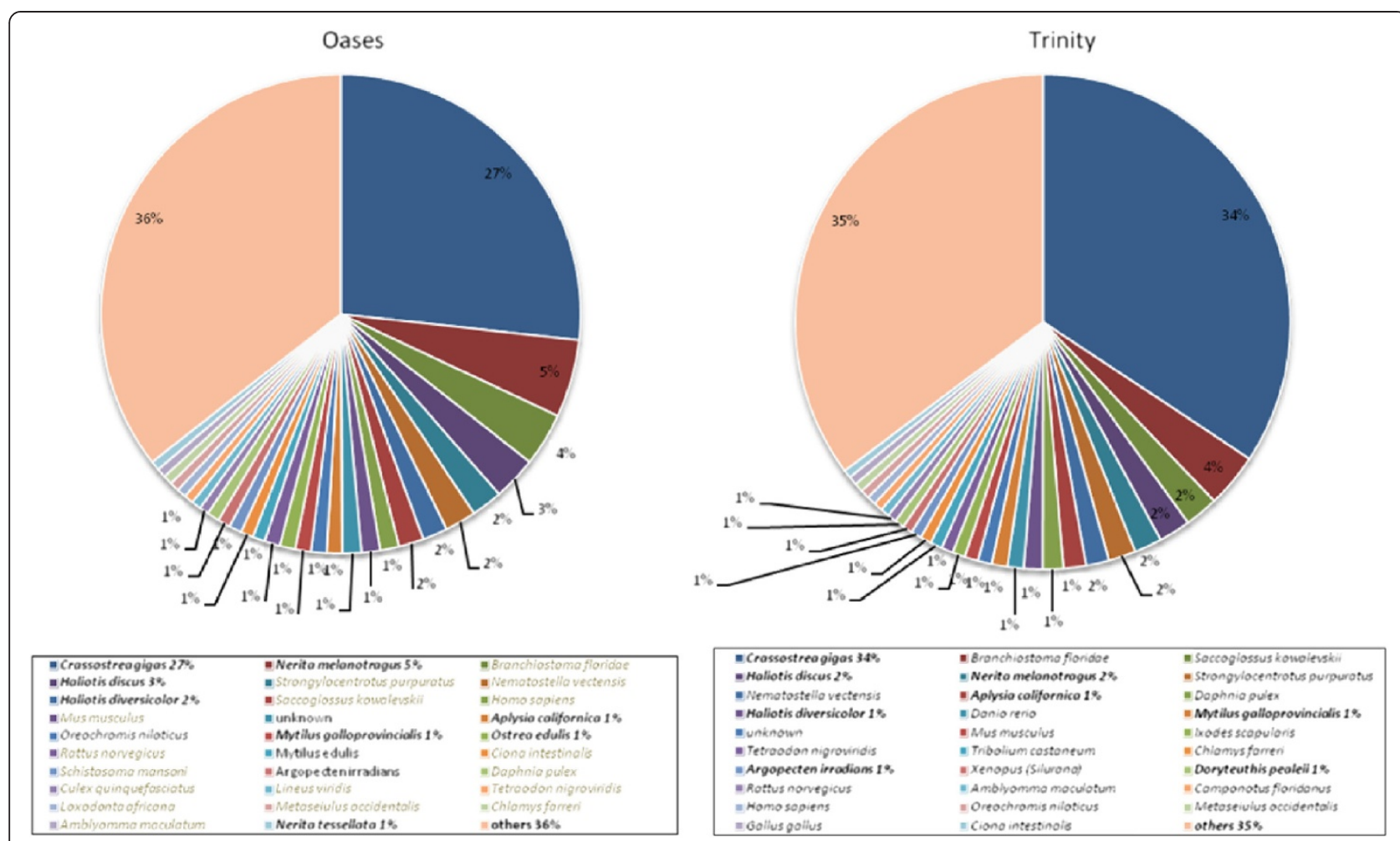

Figure 3 BLAST top hit species distribution. The 20 species most commonly represented in BLAST hits for Trinity and Oases assemblies.

performed better for different quality metrics. For example, Trinity had a longer N50, while Oases produced fewer contigs with less redundancy.

The de novo assemblies generated by both Trinity and Oases produced N50 and average contig lengths similar to many past transcriptome sequencing studies [11-13]. The N50 and average contig size of our Trinity assembly are also similar to that reported for the recently sequenced transcriptome of the common pond snail, Radix balthica [27]. In contrast, the N50 and average contig size (>1200 bp) reported for a transcriptome sequence of a different pond snail, Lymnaea stagnalis [4] are $6 \mathrm{x}$ larger than that of the Trinity assembly for our dataset. A few differences between our transcriptome assembly and that for the L. stagnalis transcriptome assembly may account for this difference. Firstly, their dataset had approximately 40x more 100 bp Illumina sequences than in our study. Secondly, the L. stagnalis study was conducted for a single tissue type, the central nervous system, while our study utilized the whole animal. These two factors may explain much of the difference in N50 and average contig length between the two studies. Therefore, it is highly likely that de novo assemblies generated with a similar amount of Ion Torrent data could result in assemblies with more comparable N50 and average contig lengths.
The blast and annotation success for both the Trinity and Oases assemblies was quite low (15-19\%). This level of annotation success is much lower than that often reported in the literature even for non-model species [25]. The level of annotation success achieved in this study, however, is in a similar range to that reported for two recently sequenced gastropod transcriptomes using short read technologies ( $R$. balthica $17 \%$ and L. stagnalis $20.1 \%)[27,4]$. One of the reasons put forward to explain the low degree of annotation success is the fact that few reference genome sequences exist for mollusc species [4]. We also hypothesise that an improvement in annotation success of gastropod species will require more representative gastropod reference genome sequences and an increase in mollusc protein sequences in public databases.

In this paper we describe an EST collection generated by Ion Torrent sequencing and de novo assembly to characterize the transcriptome of a non-model gastropod species, $N$. melanotragus. This marine gastropod is a common component of the intertidal zone on rocky substrates and distributed from Mackay (Queensland) to southern Tasmania and New Zealand. Across this large geographic distribution $N$. melanotragus spans a number of environmental gradients such as clines in water temperature, oxygen concentration and substrate type 
[16]. The environment of this species also varies temporally on a micro geographic scale between tidal cycles and consequently $N$. melanotragus is exposed to large changes in a number of environmental factors including temperature, $\mathrm{pH}$, salinity and dissolved oxygen [16]. Therefore generating genomic datasets such as in this study is crucial as we focus our research efforts towards understanding genetic and gene expression changes that have allowed this intertidal species to adapt and cope with dramatic fluctuations in environmental conditions.

\section{Conclusion}

The large number of contigs that we have annotated and functionally characterized in this study provides a first step towards a systems biology approach to physiological genomics in gastropod species. By identifying a wide variety of genes from a number of different GO classes we can now determine which genes are important for adaptation across broad environmental changes and for stress response to micro geographic environmental fluctuations. This is very important because we still know remarkably little about the physiology and evolution of many marine organisms and in particular the physiological basis of adaptation to both spatial and temporal environmental variation in intertidal zone species $[28,29]$.

\section{Availability of supporting data}

The raw sequence reads supporting the results of this article are available in the short reads archive under the accession number SRR1054996 (http://www.ncbi.nlm. nih.gov/sra/?term=SRR1054996). PCR validation gene sequences are available under the accession number KM025036 (beta-actin) and KM025037 (NADH dehydrogenase subunit 5).

\section{Additional file}

Additional file 1: Table S1. Primer sequences for $\beta$-actin and NADH dehydrogenase subunit 5. Primers were designed to validate the reliability and accuracy of our assembly and annotation. Figure S1 Visualisation of PCR products. Agarose electrophoresis gel showing two candidate genes beta-actin (A) and NADH dehydrogenase (B) (Molecular marker Hyperladder IV). Figure S2 Nerita melanotragus transcriptome functional annotation based on Trinity Blast2GO analysis. Functional annotation results indicate the relative amount of each category of contigs with protein hits. The results are summarized as follows: Biological Process (BP), Molecular Function (MF) and Cellular Component (CC). Figure S3 Nerita melanotragus transcriptome functional annotation based on Oases Blast2GO analysis. Functional annotation results indicate the relative amount of each category of contigs with protein hits. The results are summarized as follows: Biological Process (BP), Molecular Function (MF) and Cellular Component (CC)

\section{Competing interests}

The authors declare that they have no competing interests.

\section{Authors' contributions}

SA performed experimental work and sequencing analysis. PP, EG and AP designed the experiment, helped with data interpretation and provided guidance throughout the whole study. All authors have read and approve of the manuscript.

\section{Acknowledgements}

The authors of this study would like to thank the School of Biomedical Science for providing funding for this research and QUT Molecular Genetics Research Facility (MGRF) for providing lab space. Thanks are also extended to Mr. Vincent Chand for providing technical assistance during the project.

\section{Author details}

${ }^{1}$ School of Biomedical Sciences, Faculty of Health, Queensland University of Technology, GPO Box 2434, Brisbane, Qld 4001, Australia. ${ }^{2}$ School of Earth, Environmental and Biological Sciences, Science and Engineering Faculty, Queensland University of Technology, GPO Box 2434, Brisbane, Qld 4001, Australia. ${ }^{3}$ Institute for Molecular Biosciences, University of Queensland, St Lucia, Qld 4072, Australia.

Received: 5 February 2014 Accepted: 25 July 2014

Published: 1 August 2014

\section{References}

1. Hou R, Bao Z, Wang S, Su H, Li Y, Du H, Hu J, Wang S, Hu X: Transcriptome sequencing and De novo analysis for yesso scallop (Patinopecten yessoensis) using 454 GS FLX. PLoS One 2011, 6:21560.

2. Ponder WF, Lindberg DR: Towards a phylogeny of gastropod molluscs: an analysis using morphological characters. Zool J Linnean Soc 1997, 119:83-265.

3. Peterson $\mathrm{CH}$ : Recruitment overfishing in a bivalve mollusc fishery: hard clams (Mercenaria mercenaria) in North Carolina. Can J Fish Aquat SC 2002, 59:96-104.

4. Sadamoto H, Takahashi H, Okada T, Kenmoku H, Toyota M, Asakawa Y: De novo sequencing and transcriptome analysis of the central nervous system of mollusc lymnaea stagnalis by deep RNA sequencing. PLoS One 2012, 7:42546

5. Sattelle DB, Buckingham SD: Invertebrate studies and their ongoing contributions to neuroscience. Invert Neurosci 2006, 6:1-3.

6. Herpin A, Badariotti F, Rodet F, Favrel P: Molecular characterization of a new leucine-rich repeat-containing $G$ protein-coupled receptor from a bivalve mollusc: evolutionary implications. Biochim Biophys Acta Gene Struct Expr 2004, 1680:137-144.

7. Zhao X, Yu H, Kong L, Li Q: Transcriptomic responses to salinity stress in the pacific oyster Crassostrea gigas. PLoS One 2012, 7:e46244.

8. Pante E, Rohfritsch A, Becquet V, Belkhir K, Bierne N, Garcia P: SNP detection from de novo transcriptome sequencing in the bivalve macoma balthica: marker development for evolutionary studies. PLoS One 2012, 7:e52302

9. Fiedler TJ, Hudder A, MCKay SJ, Shivkumar S, Capo TR, Schmale MC, Walsh $\mathrm{PJ}$ : The transcriptome of the early life history stages of the California sea hare Aplysia californica. Comparative Biochem Physiol Part D Genomics Prot 2010, 5:165-170.

10. Feng Z-P, Zhang Z, van Kesteren RE, Straub VA, van Nierop P, Jin K, Nejatbakhsh N, Goldberg Jl, Spencer GE, Yeoman MS, Wildering W, Coorssen JR, Croll RP, Buck LT, Syed NI, Smit AB: Transcriptome analysis of the central nervous system of the mollusc Lymnaea stagnalis. BMC Genomics 2009, 10:1471.

11. Hook SE, Twine NA, Simpson SL, Spadaro DA, Moncuquet P, Wilkins MR: 454 pyrosequencing-based analysis of gene expression profiles in the amphipod Melita plumulosa: transcriptome assembly and toxicant induced changes. Aquat Toxicol 2014, article in press.

12. Schunter C, Vollmer SV, Macpherson E, Pascual M: Transcriptome analyses and differential gene expression in a non-model fish species with alternative mating tactics. Acta Vet Scand 2014, 15:167.

13. Li P, Deng W-Q, Li T-H, Song B, Shen Y-H: Illumina-based de novo transcriptome sequencing and analysis of Amanita exitialis basidiocarps. Gene 2013, 532:63-71.

14. Chiara M, Horner DS, Spada A: De novo assembly of the transcriptome of the non-model plant Streptocarpus rexii employing a novel heuristic to recover locus-specific transcript clusters. PLoS One 2013, 8:e80961. 
15. Crandall ED, Frey MA, Grosberg RK, Barber PH: Contrasting demographic history and phylogeographical patterns in two indo-pacific gastropods. Mol Ecol 2008, 17:611-626.

16. Waters JM, King TM, O'loughlin PM, Spencer HG: Phylogeographical disjunction in abundant high-dispersal littoral gastropods. Mol Ecol 2005, 14:2789-2802.

17. Wheat $\mathrm{CW}$, Vogel $\mathrm{H}$ : Transcriptome sequencing goals, assembly, and assessment. Methods Mol Biol 2011, 772:129-144.

18. Kearse M, Moir R, Wilson A, Stones-Havas S, Cheung M, Sturrock S, Buxton S, Cooper A, Markowitz S, Duran C, Thierer T, Ashton B, Meintjes P, Drummond A: Geneious Basic: An integrated and extendable desktop software platform for the organization and analysis of sequence data. Bioinformatics 2012, 28:1647-1649.

19. Zerbino DR, Birney E: Velvet: algorithms for de novo short read assembly using de bruijn graphs. Genome Res 2008, 18:821-829.

20. Schulz MH, Zerbino DR, Vingron M, Birney E: Oases: robust de novo RNAseq assembly across the dynamic range of expression levels. Bioinformatics 2012, 28:1086-1092.

21. Grabherr MG, Haas BJ, Yassour M, Levin JZ, Thompson DA, Amit I, Adiconis X, Fan L, Raychowdhury R, Zeng Q, Chen Z, Mauceli E, Hacohen N, Gnirke A, Rhind N, Di Palma F, Birren BW, Nusbaum C, Lindblad-Toh K, Friedman N, Regev A: Full-length transcriptome assembly from RNA-Seq data without a reference genome. Nat Biotechnol 2011, 29:644-652.

22. Conesa A, Götz S, García-Gómez JM, Terol J, Talón M, Robles M, Blast2GO: A universal tool for annotation, visualization and analysis in functional genomics research. Bioinformatics 2005, 21:3674-3676.

23. Sexton GJ, Frere CH, Dieters MJ, Godwin ID, Prentis PJ: Development and characterization of microsatellite loci for Khaya senegalensis (Meliaceae). Am J Bot 2010, 97:e111-e113.

24. Gibbons JG, Janson EM, Hittinger $C T$, Johnston M, Abbot P, Rokas A: Benchmarking next-generation transcriptome sequencing for functiona and evolutionary genomics. Mol Biol Evol 2009, 26:2731-2744.

25. Li R, Fan W, Tian G, Zhu H, He L, Cai J, Huang Q, Cai Q, Li B, Bai Y, Zhang Z, Zhang Y, Wang W, Li J, Wei F, Li H, Jian M, Li J, Zhang Z, Nielsen R, Li D, Gu W, Yang Z, Xuan Z, Ryder OA, Leung FC-C, Zhou Y, Cao J, Sun X, et al: The sequence and de novo assembly of the giant panda genome. Nature 2010, 463:311-317.

26. Martin JA, Wang Z: Next-generation transcriptome assembly. Nat Rev Genet 2011, 12:671-682.

27. Feldmeyer B, Wheat CW, Krezdorn N, Rotter B, Pfenninger M: Short read illumina data for the de novo assembly of a non-model snail species transcriptome (radix balthica, basommatophora, pulmonata), and a comparison of assembler performance. BMC Genomics 2011, 12:317.

28. Place SP, Menge BA, Hofmann GE: Transcriptome profiles link environmental variation and physiological response of Mytilus californianus between Pacific tides. Funct Ecol 2012, 26:144-155.

29. Chapperon C, Seuront L: Erratum: variability in the motion behaviour of intertidal gastropods: ecological and evolutionary perspectives. J Mar Biol Assoc U K 2011, 91:1717.

doi:10.1186/1756-0500-7-488

Cite this article as: Amin et al:: Assembly and annotation of a nonmodel gastropod (Nerita melanotragus) transcriptome: a comparison of De novo assemblers. BMC Research Notes 2014 7:488.

\section{Submit your next manuscript to BioMed Central and take full advantage of:}

- Convenient online submission

- Thorough peer review

- No space constraints or color figure charges

- Immediate publication on acceptance

- Inclusion in PubMed, CAS, Scopus and Google Scholar

- Research which is freely available for redistribution

Submit your manuscript at www.biomedcentral.com/submit 